\title{
Septic shock due to Pasteurella multocida bacteremia: a case report
}

\author{
Niyati Narsana* and Faria Farhat
}

\begin{abstract}
Introduction: Pasteurella is a Gram-negative coccobacillus that causes a wide spectrum of diseases in humans and is commonly transmitted from cat and dog bites. An increasing number of cats and dogs are kept as pets in American households which increases the risk of pet-related infections.

Case presentation: An 82-year-old African American woman with multiple comorbidities presented with fever, vomiting and diarrhea; she later developed septic shock requiring vasopressors and intubation. She was found to have Pasteurella multocida bacteremia. Her hospital course was complicated by a pulseless electrical activity arrest. She had exposure to her pet cat at home. We believe that a possible portal of entry was her skin; however, other possibilities such as respiratory tract could not be excluded. She was treated with imipenem-cilastatin and discharged after 25 days.

Conclusions: Studies have shown a mortality range from 7 to 31\% in Pasteurella bacteremia. Due to an increasing number of pets and high mortality of this disease, it is important to have a high suspicion for this infection, especially in elderly and immunocompromised patients.
\end{abstract}

Keywords: Bacteremia, Cat scratch, Pasteurella multocida, Septic shock

\section{Introduction}

Pasteurella multocida is small Gram-negative coccobacillus that is a component of the upper respiratory tract and gastrointestinal flora of many animals [1]. Human infections are most commonly caused by cat and dog bites. Pasteurella is the most common organism isolated from cat and dog bites [2].

According to the recent 'National Pet Owners Survey' of the American Pet Products Manufacturers Association, 42 million and 54 million US households own cats and dogs as pets respectively [3]. There are approximately 85 million cats and 77 million dogs owned as pets in America [3]. Every year, there are approximately 300,000 emergency department visits in America due to animal bites [2].

Pasteurella can cause a wide spectrum of diseases from local infections to septic shock. We present a case of septic shock in an elderly woman due to Pasteurella multocida.

\footnotetext{
* Correspondence: Niyati.K.Narsana@medstar.net

Department of Medicine, Medstar Washington Hospital Center, 110 Irving street NW, Suite 2A-50, Washington, DC 20010, USA
}

\section{Case presentation}

An 82-year-old African American woman presented to our emergency department with a 2-hour history of non-bilious, non-bloody vomiting, and one episode of loose stools. She later developed worsening shortness of breath and was found to be febrile to $39.7^{\circ} \mathrm{C}$, pulse 104 beats/minute, blood pressure $169 / 71 \mathrm{mmHg}$, respiratory rate of 18 breaths/minute and saturating $95 \%$ on room air. She was obese with body mass index 35 and her examination was significant only for some bibasilar crackles. She denied any travel history, contact with people who were ill, abdominal pain, chest pain or dysuria.

Her past medical history was significant for hypertension, hyperlipidemia, chronic obstructive pulmonary disease (COPD), coronary artery disease status post-coronary artery bypass, stroke, and breast cancer status postchemotherapy and radiation therapy. Laboratory test results on admission were remarkable for white blood cell count (WBC) of 15,500 , lactic acid of $2.5 \mathrm{mmol} / \mathrm{l}$, creatinine of $1.19 \mathrm{mg} / \mathrm{dl}$ which was her baseline, and negative troponins. An electrocardiogram showed normal sinus rhythm. Computed tomography (CT) of her abdomen-pelvis was unremarkable. Her chest X-ray

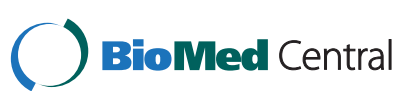


showed cardiomegaly with some pulmonary venous congestion. The suspicion for sepsis was high; however, the source was unclear at that time. Blood and urine cultures were sent, and on an empirical basis vancomycin and piperacillin-tazobactam were administered intravenously. Her lactic acid increased to $5 \mathrm{mmol} / \mathrm{l}$, creatinine to $2.28 \mathrm{mg} / \mathrm{dl}$ and WBC to 27,000 in a few hours. She became hypotensive requiring pressor support and was admitted to our intensive care unit for possible septic shock. Overnight, she had a cardiopulmonary arrest with pulseless electrical activity and there was a return of spontaneous circulation after chest compressions and epinephrine in 5 minutes. She was intubated for hypoxic respiratory failure. Her arterial blood gas showed $\mathrm{pH}$ of 7.14, partial pressure of oxygen $\left(\mathrm{pO}_{2}\right)$ of $98 \mathrm{mmHg}$, partial pressure of carbon dioxide $\left(\mathrm{pCO}_{2}\right)$ of $43 \mathrm{mmHg}$ and bicarbonate of $15 \mathrm{mmol} / \mathrm{l}$. She received alteplase and was started on heparin drip for possible pulmonary embolism. On the second day, two blood cultures grew nonmotile Gram-negative rods and vancomycin was stopped. Due to the worsening respiratory status and suspicion for ventilator-associated pneumonia and extended-spectrum beta-lactamases, piperacillin-tazobactam was switched to imipenem-cilastatin. The colonies of the organism grew on blood and chocolate agar but not on MacConkey agar. The growth on blood agar was gray and non-hemolytic (Figs. 1 and 2).

It was catalase, oxidase and indole positive and a RapID NH test (a qualitative test to identify species of Neisseria, Haemophilus and other related microorganisms isolated from humans) confirmed it as Pasteurella multocida. Her bronchial brush, sputum and urine cultures remained negative.

On careful examination, there were scratch marks and abrasions on her left leg. On further history, her daughter

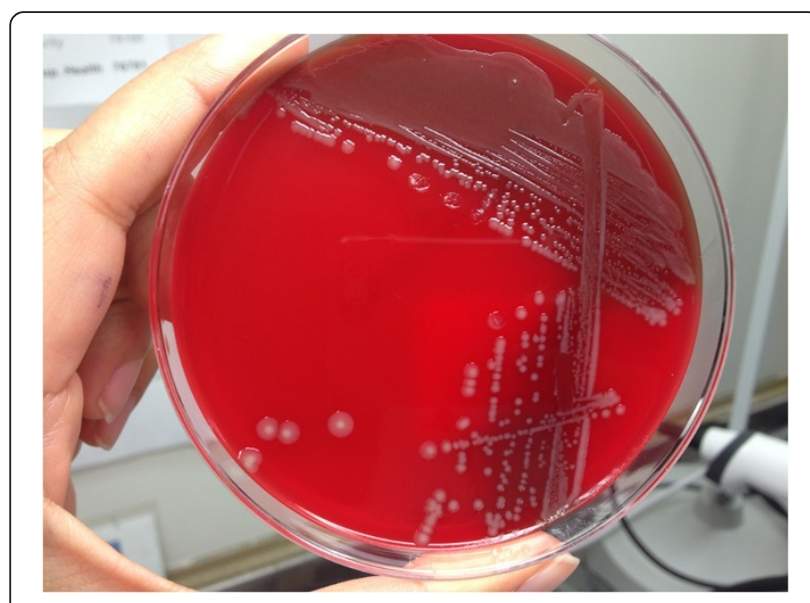

Fig. 1 Grey non-hemolytic colonies of Pasteurella multocida on blood agar. Pasturella multocida is aerobic and facultative anaerobic and grows on blood agar, chocolate agar but not on MacConkey's agar. Most isolates are oxidase, indole and catalase positive

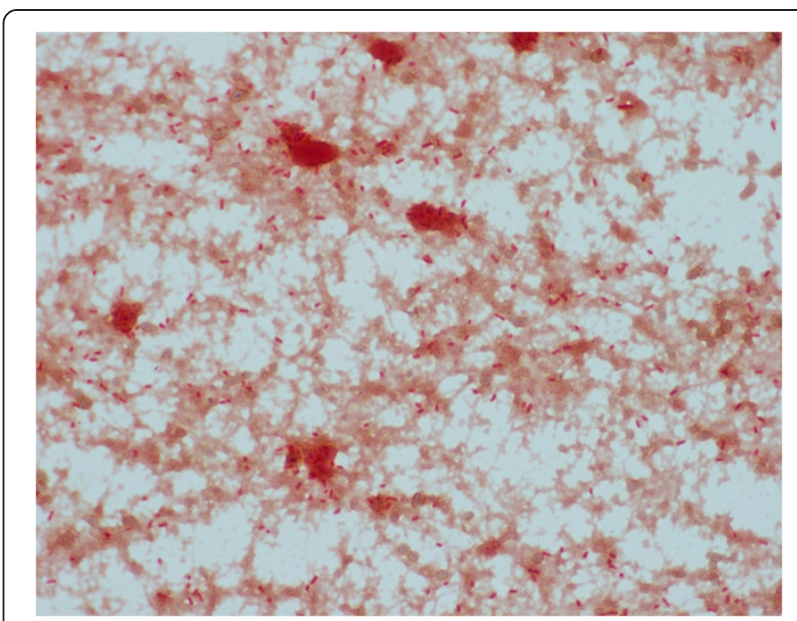

Fig. 2 Non-motile Gram-negative rods of Pasteurella. Gram-negative rods of Pasteurella as seen under the microscope

mentioned that her cat died a few days ago, and she bought another cat after that. We believe that the most likely route of transmission of Pasteurella infection was through the skin; however, another route could not be ruled out. She was eventually weaned off the pressors and was extubated after 7 days. The repeat blood cultures were negative. Her hospital course was complicated by hematemesis leading to aspiration, repeat cardiopulmonary arrest and re-intubation. At that time, she received steroids for possible COPD exacerbation. She also developed Clostridium difficile colitis, and was treated with oral vancomycin for 14 days. She was extubated successfully after 3 days, and was treated with imipenem-cilastatin for a total of 19 days for Pasteurella bacteremia and possible aspiration pneumonia. She was discharged after 25 days of hospitalization to a subacute rehabilitation center.

\section{Discussion}

Pasteurella infections in humans commonly result from contact with animals such as cats, dogs, swine, lions, panthers, horses, rats, rabbits, and wolves [4-6]. It is a common commensal organism found in the oropharynx. Cats and dogs have a colonization rate of approximately 70 to $90 \%$ and 20 to $50 \%$ respectively $[4,6,7]$. Animal bites and scratches are the most common modes of acquisition of infection in humans. Cases of infection without any animal exposure have also been reported $[4,8]$.

Pasteurella is a non-motile aerobe and facultative anaerobe, which grows on chocolate and blood agar, but not on MacConkey agar. Pasteurella multocida does not cause hemolysis on blood agar, and grows in carbon dioxide-rich medium at $37^{\circ} \mathrm{C}[4,5]$.

It can involve skin and soft tissue, bone and joint, upper and lower respiratory tract, and cause more severe infections such as meningitis, bacteremia, endocarditis and peritonitis. Local cutaneous infections are the most 
common $[5,9,10]$. In a study by Escande and Lion, bacteremia was found in 11\% of 958 cases of Pasteurella infections [10]. In a study by Ebright et al. at Detroit Medical Center, $7.8 \%$ of 179 patients had positive blood cultures [9]. The various sources of isolation of this organism reported in the literature are local wounds, sputum, bronchoalveolar lavage, cerebrospinal, pleural, ascitic and joint fluid $[5,9,10]$. There are many cases where the source of bacteremia is unknown $[10,11]$. Our patient had the organism isolated only from her blood and the possible route of entry was a cat scratch. However, other possible routes of entry such as the respiratory tract could not be confirmed as bronchial brush cultures were negative.

Pasteurella infections are more common in elderly patients with underlying chronic diseases such as diabetes mellitus, hypertension, cardiac disease, acquired immunodeficiency syndrome (AIDS), malignancies, COPD, cirrhosis and dialysis $[9,10,12]$. The study by Escande and Lion showed $70 \%$ of systemic infections in ages $>50$ years and $21 \%$ in ages 20 to 50 years [10]. Our patient had multiple comorbidities including hypertension, hyperlipidemia, COPD and coronary artery disease which made her more susceptible to developing this severe infection.

Most of the studies have shown susceptibility of Pasteurella multocida to penicillins, beta-lactams, carbapenems, second and third generation cephalosporins and tetracyclines [5, 12-14]. Severe systemic infections are treated with intravenous antibiotics and local infections with oral agents $[9,10]$. Studies have shown a mortality ranging from 7 to $31 \%$ in patients with Pasteurella bacteremia [9-11].

\section{Conclusions}

There is an increasing number of pets in American households leading to increased exposure to animals. This can increase the risk of developing infections, especially in the elderly and immunocompromised population. It is important to maintain appropriate hygiene to prevent transmission of infection from pets. Given the high mortality of this disease and increasing number of pets, it is important to have a high suspicion for pet-related infections such as Pasteurella multocida.

\section{Consent}

Written informed consent was obtained from the patient for publication of this case report and accompanying images. A copy of the written consent is available for review by the Editor-in-Chief of this journal.

\section{Abbreviations}

COPD: Chronic obstructive pulmonary disease; WBC: White blood cell count.

\section{Competing interests}

The authors declare that they have no competing interests.

\section{Authors' contributions}

NN collected the information and was the major contributor of the manuscript. FF and NN were involved in patient care. FF contributed to revising the manuscript. All authors read and approved the final manuscript.

Received: 8 December 2014 Accepted: 23 June 2015

Published online: 11 July 2015

\section{References}

1. Fajfar-Whetstone CJ. Carol. Pasteurella multocida septicemia and subsequent pasteurella dagmatis septicemia in a diabetic patient. J Clin Microbiol. 1995;33(1):202-04.

2. Christenson ES, Ahmed HM, CM D. Pasteurella multocida infection in solid organ transplantation. Lancet Infect Dis. 2015;15(2):235-40.

3. American Pet Products Association: http://www.americanpetproducts.org/ press_industrytrends.asp (2015). Accessed 18 May 2015.

4. Klein NC, Cunha BA. Pasteurella multocida pneumonia. Semin Respir Infect. 1997;12(1):54-6.

5. Weber DJ, Wolfson JS, Swartz MN, Hooper DC. Pasteurella multocida infections. Medicine. 1984;63(3):133-54.

6. Morris JT, Kenneth MC. Bacteremia due to pasteurella multocida. South Med J. 1992;85(4):442-43.

7. Westling K, Bygdeman S, Engkvist O, Jorup-Ronstrom C. LETTER: pasteurella multocida infection following cat bites in humans. J Infect. 2000;40(1):97-8.

8. Ruiz-Irastorza G, Garea C, Alonso JJ, Hernandez JL, Aguirrebengoa K, Alonso J, et al. Septic shock due to pasteurella multocida subspecies multocida in a previously healthy woman. Clin Infect Dis. 1995;21(1):232-34.

9. Ebright J, Frey A, Fairfax M. Pasteurella multocida infections and bacteremia. Infect Dis Clin Pract. 2009;17(2):102-04.

10. Escande F, Lion C. Epidemiology of human infections by Pasteurella and related groups in France. Zentralblatt Für Bakteriologie. 1993;279(1):131-39.

11. Raffi F, Jacques B, Baron D, Drugeon HB, Nicolas F, Courtieu AL. Pasteurella multocida bacteremia: report of thirteen cases over twelve years and review of the literature. Scand J Infect Dis. 1987;19(4):385-93.

12. Christidou A, Maraki S, Gitti Z, Tselentis Y. Review of pasteurella multocida infections over a twelve-year period in a tertiary care hospital. Am J Infect Dis. 2005:1(2):107-10.

13. Lion C, Conroy M, Carpentier A, Lozniewski A. Antimicrobial susceptibilities of pasteurella strains isolated from humans. Int J Antimicrob Agents. 2006;27(4):290-93.

14. Adler AC, Cestero C. Septic shock from Pasteurella multocida following a cat bite.: case report and review of literature. Conn Med. 2011;75(10):603-5.

\section{Submit your next manuscript to BioMed Central and take full advantage of:}

- Convenient online submission

- Thorough peer review

- No space constraints or color figure charges

- Immediate publication on acceptance

- Inclusion in PubMed, CAS, Scopus and Google Scholar

- Research which is freely available for redistribution 